\title{
Correction to: Numerical Methods for Energy Applications
}

Naser Mahdavi Tabatabaei and Nicu Bizon

\section{Correction to:}

N. Mahdavi Tabatabaei and N. Bizon (eds.), Numerical Methods for Energy Applications, Power Systems, https://doi.org/10.1007/978-3-030-62191-9

The original version of these chapters "Numerical Methods in Selecting Location of Distributed Generation in Energy Network" and "Numerical Methods for Power System Analysis with FACTS Devices Applications" are inadvertently published with branch name after the institution name. It has now been corrected. 